\title{
RIGIDITY OF THE HAMBURGER AND STIELTJES MOMENT SEQUENCES
}

\author{
ALEXANDER DYACHENKO \\ TU-Berlin, Institut für Mathematik, Sekr. MA 4-2, \\ Straße des 17. Juni 136, 10623 Berlin, Germany.
}

\begin{abstract}
This paper aims at finding conditions on a Hamburger or Stieltjes moment sequence, under which the change of at most a finite number of its entries produces another sequence of the same type. It turns out that a moment sequence allows all small enough variations of this kind precisely when it is indeterminate. We also show that a determinate moment sequence has the finite index of determinacy if and only if the corresponding finite number of its entries can be changed in a certain way.
\end{abstract}

\section{BRIEF INTRODUCTION}

The classical moment problems by Stieltjes and Hamburger play an important role in many branches of mathematics. They consist in finding a distribution of masses (a positive measure) based on a sequence of the real numbers which are called moments. Sequences of the moments can be characterized by positivity of the related Hankel quadratic forms, and the induced interrelationship between the entries is relatively strong. Indeed, although increasing the leading (i.e. zeroth) moment is always possible, other changes of finitely many moments turn to be impossible for many moment sequences: here we call such sequences "rigid". At the same time, "nonrigid" moment sequences may allow more or less free variations of their entries, and the criterion for this freedom seems to be absent in literature.

We use the so-called index of determinacy to express the tightness of the conditions arising from positivity of the Hankel forms. Our first goal is to describe its connection to the rigidity of moment sequences. In particular, we show that this index determines the minimal number of leading moments which can be varied.

The second goal is to find out whether or not these variations can be arbitrary. It turns out that indeterminate moment sequences survive all small enough changes of finitely many entries. At the same time, determinate sequences may survive only specific variations: one of the moments allows all small changes if and only if the corresponding moment problem is indeterminate.

The author is very grateful to Alan Sokal and Christian Berg for their remarks, as well as for introducing the problem and the initial idea. This research was supported by the Einstein Foundation Berlin.

\section{DEFINITIONS AND BASIC FACTS}

For introducing rigorous statements and proofs, we need certain basic facts and definitions, most of which can be found in the classical books $[1,9]$.

E-mail address: diachenko@sfedu.ru, dyachenk@math.tu-berlin.de.

Date: 1st December 2017.

2010 Mathematics Subject Classification. 30E05 - 44A60 - 42C05.

Key words and phrases. Moment problems · Index of determinacy. 
Let $\mathfrak{h}=\left(c_{i}\right)_{i=0}^{\infty}=\left(c_{0}, c_{1}, c_{2}, \ldots\right)$ be a sequence of the Hamburger moments, that is let there exist some (positive) measure ${ }^{1} d \mu(x)$ on the real line such that

$$
c_{i}=\int_{-\infty}^{\infty} x^{i} d \mu(x) \text { for all } i=0,1, \ldots
$$

The work [6] established that $\mathfrak{h}$ is a Hamburger moment sequence if and only if all quadratic forms

$$
\sum_{i, j=0}^{p} c_{i+j} x_{i} x_{j}, \quad p=0,1,2, \ldots
$$

are positive semidefinite. Given a Hamburger moment sequence $\mathfrak{h}$, we write $\mathfrak{h} \in \operatorname{Det}_{H}$ if it is determinate, i.e. if the measure $d \mu(x)$ is uniquely determined by (1), or $\mathfrak{h} \in \operatorname{Indet}_{H}$ otherwise.

Note that the trimmed sequence of moments $\left(c_{i}\right)_{i=2 n}^{\infty}$ corresponds for each $n=1,2, \ldots$ to the measure $x^{2 n} d \mu(x)$. If $\left(c_{i}\right)_{i=2 n}^{\infty}$ determines the unique measure $x^{2 n} d \mu(x)$, then $d \mu(x)$ is uniquely determined outside the origin, while the atom at the origin is fixed by the moment $c_{0}$. That is, prefixing a moment sequence with a pair of new entries only means introducing additional constraints, which cannot make the problem "less definite":

Lemma 1. If $\mathfrak{h}=\left(c_{i}\right)_{i=0}^{\infty} \in \operatorname{Indet}_{H}$, then $\left(c_{i}\right)_{i=2 n}^{\infty} \in \operatorname{Indet}_{H}$ for $n=1,2, \ldots$ Accordingly, if $\left(c_{i}\right)_{i=2 n}^{\infty} \in$ $\operatorname{Det}_{H}$ for some positive integer $n$, then $\mathfrak{h} \in \operatorname{Det}_{H}$.

This lemma allows introducing the index of determinacy (see [2])

$$
\operatorname{ind}_{0}(\mathfrak{h}):=\sup \left\{n \in \mathbb{Z}_{\geq 0}:\left(c_{i}\right)_{i=2 n}^{\infty} \in \operatorname{Det}_{H}\right\}
$$

of a determinate Hamburger sequence $\mathfrak{h}$. In other words, $\operatorname{ind}_{0}(\mathfrak{h})$ is the minimal nonnegative number such that the measure $x^{2 n} d \mu(x)$ with an integer $n$ is uniquely determined by its moments as soon as $0 \leq n \leq \operatorname{ind}_{0}(\mathfrak{h})$ and not uniquely determined for all $n>\operatorname{ind}_{0}(\mathfrak{h})$. The work [2] introduces the index of determinacy through the measure, which allows calculating it with respect to other points than the origin. Both definitions coincide for the origin, and ours better fits to the current study. It turns out that, if $\operatorname{ind}_{0} \mathfrak{h}<\infty$, then the corresponding measure $\mu(x)$ is discrete. Another result of [2] is that the indices for distinct points can differ by at most 1.

Each distribution of masses $d \mu(z)$ satisfying (1) determines a unique ${ }^{2}$ mapping of the upper half-plane $\{z \in \mathbb{C}: \Im z>0\}$ into itself by the formula [1, p. 95, Thm. 3.2.1]

$$
\int_{-\infty}^{\infty} \frac{d \mu(x)}{x-z}
$$

where the integral has the asymptotic expansion

$$
-\frac{c_{0}}{z^{1}}-\frac{c_{1}}{z^{2}}-\frac{c_{2}}{z^{3}}-\cdots \quad \text { as } \quad z \rightarrow+\infty \cdot i .
$$

This fact yields a characterization of determinate Hamburger moment sequences: the related asymptotic series (3) represents only one mapping of the upper half-plane into itself.

Remark 2. As follows from Kronecker's studies, singularity of any of the positive semidefinite forms (2) is equivalent to that the support of the corresponding measure $d \mu(x)$ has finitely many points. Supports of such measures are compact, and thus the related index of determinacy is always infinite: the series (3) turn to the Taylor series at infinity. Theorem 4 and Corollary 5 in Section 3 show that the moment sequences of such measures are rigid. Sections $\underline{4}-\underline{6}$ only deal with nonrigid sequences, so the corresponding forms (2) are necessarily positive definite therein.

\footnotetext{
${ }^{1}$ We introduce a slight abuse in the notation by using the differential $d \mu(x)$ of a non-decreasing function $\mu(x)$ for denoting the related measure.

${ }^{2}$ The uniqueness follows from the Stieltjes-Perron inversion formula [1, pp. 123-126]
} 
The sequence $\mathfrak{s}=\left(s_{i}\right)_{i=0}^{\infty}$ is called the Stieltjes moment sequence provided that there exists some measure $d \sigma(x)$ on the positive semi-axis such that

$$
s_{i}=\int_{0}^{\infty} x^{i} d \sigma(x) \text { for all } i=0,1, \ldots
$$

The set of Stieltjes moment sequences is hence embedded in the set of Hamburger moment sequences. Moreover, the sequence $\mathfrak{s}$ is a Stieltjes moment sequence if and only if both sequences $\mathfrak{s}$ and $\left(s_{i}\right)_{i=1}^{\infty}$ are Hamburger moment sequences. We write $\mathfrak{s} \in \operatorname{Det}_{S}$ if $\mathfrak{s}$ is determinate, i.e. if the measure $d \sigma(x)$ is uniquely determined by (4), or $\mathfrak{s} \in$ Indet $_{S}$ otherwise. Although Indet $_{S}$ contains only those Stieltjes sequences that belong to $\operatorname{Indet}_{H}$, Hamburger proved [7] that $\operatorname{Det}_{S} \cap \operatorname{Indet}_{H}$ is non-empty and gave a description of this set. Moreover, the inclusion $\mathfrak{s} \in \operatorname{Det}_{S} \cap \operatorname{Indet}_{H} \operatorname{implies}$ that the corresponding unique Stieltjes measure has an atom at the origin, see [3]. By analogy with Lemma 1 we have:

Lemma 3. If $\mathfrak{s} \in \operatorname{Indet}_{S}$, then $\left(s_{i}\right)_{i=n}^{\infty} \in \operatorname{Indet}_{S} \subset \operatorname{Indet}_{H}$ for $n=1,2, \ldots$ If $\left(s_{i}\right)_{i=n}^{\infty} \in \operatorname{Det}_{S}$ for some positive integer $n$, then $\mathfrak{s} \in \operatorname{Det}_{S}$.

For determinate Stieltjes moment sequences, the index of determinacy is defined (see [3]) by

$$
\operatorname{ind}(\mathfrak{s}):=\sup \left\{n \in \mathbb{Z}_{\geq 0}:\left(s_{i}\right)_{i=n}^{\infty} \in \operatorname{Det}_{s}\right\} \text {. }
$$

Accordingly, the measure $x^{n} d \sigma(x)$ is the only one corresponding to $\left(s_{i}\right)_{i=n}^{\infty}$ when $n \leq \operatorname{ind}(\mathfrak{s})$, and there are infinitely many measures corresponding to $\left(s_{i}\right)_{i=n}^{\infty}$ when $n>\operatorname{ind}(\mathfrak{s})$.

The Hamburger moment sequence $\mathfrak{h}$ is called symmetric if all odd moments $c_{2 n+1}, n \in \mathbb{Z}_{\geq 0}$, are equal to zero. The corresponding measure $d \mu(x)$ is not necessarily symmetric (i.e. even), but the odd part of its distribution function $\frac{1}{2}(\mu(x)-\mu(-x))$ gives a symmetric measure corresponding to the symmetric moment sequence $\mathfrak{h}$. In particular, the measure $d \mu(x)$ is necessarily symmetric if $\mathfrak{h} \in \operatorname{Det}_{H}$, and there are infinitely many symmetric solutions to the moment problem (1)

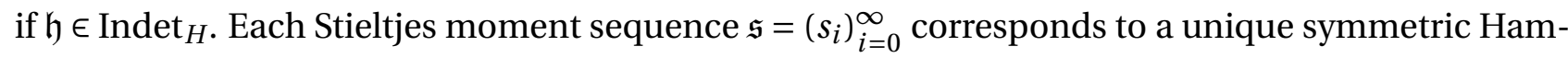
burger moment sequence $\left(s_{0}, 0, s_{1}, 0, s_{2}, 0, \ldots\right)$ and conversely: if $\left(s_{0}, 0, s_{1}, 0, s_{2}, 0, \ldots\right)$ is a symmetric Hamburger moment sequence, then $\mathfrak{s}$ is a Stieltjes moment sequence. Moreover, these sequences $\mathfrak{s}$ and $\left(s_{0}, 0, s_{1}, 0, s_{2}, 0, \ldots\right)$ are simultaneously determinate, see [4].

\section{3. “RIGIDITY” AND "NONRIGIDITY” OF MOMENT SEQUENCES}

Theorem 4. Given some positive integer n, let

$$
\mathfrak{h}=\left(c_{0}, c_{1}, \ldots, c_{2 n-1}, c_{2 n}, c_{2 n+1}, \ldots\right) \text { and } \mathfrak{t}:=\left(c_{0}^{*}, c_{1}^{*}, \ldots, c_{2 n-1}^{*}, c_{2 n}, c_{2 n+1}, \ldots\right)
$$

be two Hamburger moment sequences which differ in at most $2 n$ leading entries. If $\left(c_{i}\right)_{i=2 n}^{\infty} \in \operatorname{Det}_{H}$, then $\mathfrak{h}$ and $\mathfrak{t}$ coincide up to their leading entries $c_{0}$ and $c_{0}^{*}$.

In other words, if $\mathfrak{h} \in \operatorname{Det}_{H}$ and $\operatorname{ind}_{0}(\mathfrak{h}) \geq n$, then necessarily

$$
c_{1}^{*}=c_{1}, \quad c_{2}^{*}=c_{2}, \quad \ldots, \quad c_{2 n-1}^{*}=c_{2 n-1} .
$$

Proof. If $\left(c_{i}\right)_{i=2 n}^{\infty} \in \operatorname{Det}_{H}$, then Lemma 1 yields $\mathfrak{h}, \mathfrak{t} \in \operatorname{Det}_{H}$; thus, there exist two uniquely determined measures $d \mu(x)$ and $d v(x)$ corresponding to, resp., $\mathfrak{h}$ and $\mathfrak{t}$. Moreover, due to $x^{2 n} d \mu(x)=x^{2 n} d v(x)$ these measures can differ only by a concentrated mass at the origin: $d \mu(x)=d v(x)+M \cdot \delta(x) d x$, where $M \in \mathbb{R}$. Therefore, $c_{0}=M+c_{0}^{*}$, while the remaining entries in $\mathfrak{h}$ and $\mathfrak{t}$ coincide.

Considering symmetric Hamburger moment sequences immediately gives:

Corollary 5. Let $n$ be some positive integer, let also

$$
\mathfrak{s}=\left(s_{0}, s_{1}, \ldots, s_{n-1}, s_{n}, s_{n+1}, \ldots\right) \quad \text { and } \quad \mathfrak{t}:=\left(s_{0}^{*}, s_{1}^{*}, \ldots, s_{n-1}^{*}, s_{n}, s_{n+1}, \ldots\right)
$$


be two Stieltjes moment sequences which differ in at most $n$ leading entries. If $\left(s_{i}\right)_{i=n}^{\infty} \in \operatorname{Det}_{S}$, then $\mathfrak{s}$ and $\mathrm{t}$ coincide up to their leading entries $s_{0}$ and $s_{0}^{*}$.

In other words, if $\mathfrak{s} \in \operatorname{Det}_{S}$ and $\operatorname{ind}(\mathfrak{s}) \geq n$, then necessarily

$$
s_{1}^{*}=s_{1}, \quad s_{2}^{*}=s_{2}, \quad \ldots, \quad s_{n-1}^{*}=s_{n-1} .
$$

Theorem $\underline{4}$ shows that the Hamburger moment sequences with infinite index of determinacy are rigid. The next theorem gives the contrary for indeterminate sequences and for determinate sequences with a finite index of determinacy.

Theorem 6. Suppose that $\mathfrak{h}=\left(c_{i}\right)_{i=0}^{\infty} \in \operatorname{Indet}_{H}$ or $\operatorname{ind}_{0}(\mathfrak{h})<n$ for some positive integer $n$. Then, for arbitrary real numbers $c_{1}^{*}, c_{3}^{*}, \ldots, c_{2 n-1}^{*}$ and for any $c_{2 n}^{*}$ such that $\left|c_{2 n}^{*}-c_{2 n}\right|<\varepsilon$ with a small enough $\varepsilon$, there exists another Hamburger moment sequence

$$
\mathfrak{t}:=\left(c_{0}^{*}, c_{1}^{*}, \ldots, c_{2 n-1}^{*}, c_{2 n}^{*}, c_{2 n+1}, \ldots\right) \in \operatorname{Indet}_{H},
$$

where each of the even moments $c_{2 i}^{*}, i=0,1, \ldots, n-1$, can be chosen in certain limits. More specifically, $c_{2 i}^{*} \in\left(b_{2 i},+\infty\right)$, where $b_{2 i}>0$ depends on the values of $c_{2 i+1}^{*}, c_{2 i+2}^{*}, \ldots, c_{2 n-1}^{*}, c_{2 n}, c_{2 n+1}, \ldots$

See the proofs in Section 5. Observe that, like indeterminate Hamburger moment sequences, the determinate sequences with $\operatorname{ind}_{0}(\mathfrak{h})=0$ allow changing any finite number of leading entries. The correspondence between symmetric and Stieltjes moment sequences induces the following fact:

Corollary 7. Let $\mathfrak{s}=\left(s_{i}\right)_{i=0}^{\infty} \in \operatorname{Indet}_{S}$ or $\operatorname{ind}(\mathfrak{s}) \leq n$. Then there is the Stieltjes moment sequence

$$
\mathfrak{t}:=\left(s_{0}^{*}, s_{1}^{*}, \ldots, s_{n-1}^{*}, s_{n}, s_{n+1}, \ldots\right) \in \operatorname{Indet}_{S},
$$

such that the moments $s_{i}^{*}, i=0,1, \ldots, n-1$, can be set to any numbers within certain limits. More specifically, $s_{i}^{*} \in\left(b_{i},+\infty\right)$, where $b_{i}>0$ depends on the values of $s_{i+1}^{*}, s_{i+2}^{*}, \ldots, s_{n-1}^{*}, s_{n}, s_{n+1}, \ldots$

\section{A STRONGER VERSION OF THE PROBLEM}

The stronger version of the initial problem is to determine under which conditions "nonrigid" sequences allow arbitrary perturbations of the entries provided that the perturbations are small enough. Here we introduce a few extensions in this direction of the facts stated in the previous section.

It follows directly from the definition (1) that, if $\mathfrak{h}$ and $\mathfrak{t}$ are two Hamburger sequences corresponding, respectively, to the measures $d \mu(x)$ and $d v(x)$, then their convex combination $\eta \mathfrak{h}+(1-\eta) \mathfrak{t}$, where $0<\eta<1$, is the Hamburger sequence which corresponds to the measure $\eta d \mu(x)+(1-$ $\eta) d v(x)$. The simplest example that a sum of two determinate sequences may be indeterminate can be found in the proof of Lemma 12 , where one of the sequences is induced by the Dirac measure $\delta(x) d x$, see Section 5 . However, if at least one of the sequences $\mathfrak{h}$ and $\mathfrak{t}$ is indeterminate, then their convex combination $\eta \mathfrak{h}+(1-\eta) \mathfrak{t}$ must also be indeterminate: at least one of the summands in $\eta d \mu(x)+(1-\eta) d v(x)$ is not determined uniquely. Trimming leading entries of determinate sequences with finite indices immediately yields:

Lemma 8. Let $\mathfrak{h}$ and $\mathfrak{t}$ be two Hamburger moment sequences and let $0<\eta<1$. Then

$$
\operatorname{ind}_{0}(\eta \mathfrak{h}+(1-\eta) \mathfrak{t}) \leq \min \left\{\operatorname{ind}_{0}(\mathfrak{h}), \operatorname{ind}_{0}(\mathfrak{t})\right\} .
$$

Let the moment sequences $\mathfrak{h}$ and $\mathfrak{t}$ be as in Theorem $\underline{6}$ such that $\mathfrak{t}$ is indeterminate, then the sequence $(1-\varepsilon) \mathfrak{h}+\varepsilon \mathfrak{t}$ is also indeterminate for each $\varepsilon \in(0,1)$. At the same time, this latter sequence can be as close to $\mathfrak{h}$ as we want; therefore: 
Corollary 9. Every neighbourhood ${ }^{3}$ of each determinate Hamburger or Stieltjes moment sequence of a finite index contains an indeterminate moment sequence of the same type, which only differs in a finite number of entries.

The indeterminate sequences allow changing their entries quite freely:

Theorem 10. If a Stieltjes or Hamburger moment sequence is indeterminate, then any small enough variation of a finite number of its entries gives an indeterminate moment sequence of the same type.

This is however not true for determinate sequences, even if the index of determinacy is finite:

Theorem 11. Let $\mathfrak{h}=\left(c_{0}, c_{1}, c_{2}, \ldots\right)$ be a Hamburger or Stieltjes moment sequence, and let $m>0$ be an integer number. Then the sequence $\mathfrak{h}$ is necessarily indeterminate $\left(\mathfrak{h} \in \operatorname{Indet}_{H}\right.$ or, resp., $\left.\mathfrak{h} \in \operatorname{Indet}_{S}\right)$, if all small enough variations of the entry $c_{m}$ that change no other entries $c_{n}$ with $n \neq m$ result in moment sequences of the same type.

Let a determinate moment sequence $\mathfrak{h}=\left(c_{i}\right)_{i=0}^{\infty}$ have a finite index $\operatorname{ind}_{0}(\mathfrak{h})=: m$. After comparison of the last theorem with Theorem $\underline{6}$ and Corollary $\underline{9}$, one can ask whether it is possible to change a single moment of $\mathfrak{h}$ provided that all other entries are fixed. This question lies outside the scope of the present work, the answer however must depend on the sequence.

To illustrate, let us consider the basic case. By Theorem 6 and Corollary 9 , we may change the entries $c_{0}, \ldots, c_{2 m-1}, c_{2 m}$ in certain limits and keep $c_{2 m+1}, c_{2 m+2}, \ldots$ At the same time, the entry $c_{2 m}$ can only be increased, since $\left(c_{i}\right)_{i=2 m}^{\infty} \in \operatorname{Det}_{H}$ and the corresponding measure has no atom at the origin. More specifically, the point $\left(c_{2 m+1}, c_{2 m}\right)$ lies on the border of the parabolic region introduced in Lemma 12. That point cannot leave the region (in particular, $c_{2 m}$ cannot decrease), otherwise $\mathfrak{h}$ is no longer a Hamburger moment sequence. Now, if $\left(c_{2 m+1}, c_{2 m}\right)$ corresponds to the minimal value of $c_{2 m}$, then changing $c_{2 m+1}$ is impossible on condition that all other entries are fixed. When the value of $c_{2 m}$ is not minimal, the moment $c_{2 m+1}$ can be either increased or decreased depending on which part of the border of the involved parabolic region the point $\left(c_{2 m+1}, c_{2 m}\right)$ belongs to.

\section{Proofs of Theorems $\underline{6}$ AND $\underline{10}$}

We rely on the following fact (see its proof below):

Lemma 12. Let $\mathfrak{h}=\left(c_{0}, c_{1}, \ldots\right) \in \operatorname{Indet}_{H}$. Then there exists another Hamburger moment sequence

$$
\mathfrak{t}:=\left(c_{-2}, c_{-1}, c_{0}, c_{1}, \ldots\right)
$$

for any given real number $c_{-1}$. Moreover, $c_{-2}$ then can be set to any number satisfying

$$
c_{-2} \geq c_{-1}^{2} \sum_{k=0}^{\infty} P_{k}^{2}(0)+\sum_{k=0}^{\infty} Q_{k}^{2}(0)+2 c_{-1} \sum_{k=0}^{\infty} P_{k}(0) Q_{k}(0),
$$

here $P_{k}(z)$ is the $k$ th orthonormal polynomial related to $h$ and $Q_{k}(z)$ is the corresponding polynomial of the second kind. This inequality is strict if and only if the new sequence $t$ is indeterminate.

Remark 13. It is noteworthy that the right hand side of the last inequality for $c_{-2}$ reaches its minimum

$$
\begin{aligned}
& c_{-2}^{*}=\sum_{k=0}^{\infty} Q_{k}^{2}(0)-\rho(0)\left(\sum_{k=0}^{\infty} P_{k}(0) Q_{k}(0)\right)^{2} \text { when } c_{-1} \text { turns to } \\
& c_{-1}^{*}=-\frac{\sum_{k=0}^{\infty} P_{k}(0) Q_{k}(0)}{\sum_{k=0}^{\infty} P_{k}^{2}(0)}=-\rho(0) \sum_{k=0}^{\infty} P_{k}(0) Q_{k}(0)
\end{aligned}
$$

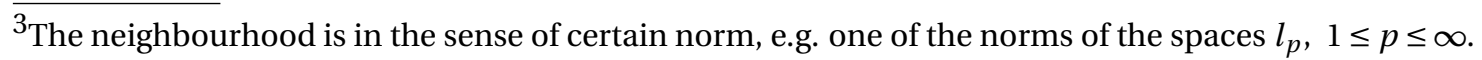


where $\rho(0)$ stands for the maximal mass that can be put at the origin among all distributions corresponding to $\mathfrak{h}$ (the related measure is labelled in the proof below by $d \mu(x ; \infty)$ ).

In the terms of the expressions (6) and (10), the minimal value of $c_{-2}$ can be expressed as

$$
c_{-2}^{*}=a^{\prime}(0)-\frac{b^{\prime}(0) c^{\prime}(0)}{d^{\prime}(0)} \text { for } c_{-1}^{*}=-\frac{b^{\prime}(0)+c^{\prime}(0)}{2 d^{\prime}(0)}=-\frac{b^{\prime}(0)}{d^{\prime}(0)} .
$$

Another option is that these formulae can be expressed through the limits of certain determinants built of the moments, see e.g. [8, p. 181] or [9, pp. 66-67]. Limits of similar determinants appear in our polynomial $p_{*}(\gamma)$ from the proof of Lemma 15 for $m=1$ : the condition $p_{*}(\gamma) \geq 0$ is, in fact, the analogue of (모).

When $\mathfrak{h}=\left(c_{i}\right)_{i=0}^{\infty} \in$ Indet $_{H}$, there exists the corresponding Nevanlinna parameterization (see e.g. [1, Ch. II \$4]) - four real entire functions $a(z), b(z), c(z), d(z)$ of at most minimal type of exponential order which satisfy the formula ${ }^{4}$

$$
m(z ; t):=\int_{-\infty}^{\infty} \frac{d \mu(x ; t)}{x-z}=-\frac{a(z)-t c(z)}{b(z)-t d(z)} \quad\left(m(z ; \infty)=-\frac{c(z)}{d(z)}\right)
$$

with the parameter $t$ running over $\mathbb{R} \cup\{\infty\}$. Each of the measures $d \mu(x ; t)$ solves the moment problem (1), that is

$$
c_{i}=\int_{-\infty}^{\infty} x^{i} d \mu(x ; t) \text { for all } i=0,1, \ldots
$$

independently of $t \in \mathbb{R} \cup\{\infty\}$. These measures are called $N$-extremal, because the polynomials are dense in the spaces $L_{2}(\mu(\cdot ; t))$. The whole set of the solutions of the moment problem (1) comes from substituting $(-t)$ in ( $\underline{6})$ by all mappings of the upper half-plane into itself. At the origin, these four functions satisfy

$$
b(0)=-1, \quad c(0)=1 \quad \text { and } \quad a(0)=d(0)=0 .
$$

Proof of Lemma 12. Suppose that $\mathfrak{h} \in \operatorname{Indet}_{H}$ and the corresponding N-extremal measure $d \mu(x ; t)$ is as in (6). We confine ourselves with finite real values of the parameter $t$. Under this condition, the support of $d \mu(x ; t)$ has no points in a neighbourhood of the origin, i.e. if $|t|<t_{\max }$, then $(-\epsilon, \epsilon) \notin$ supp $d \mu(x ; t)$ for some $\epsilon>0$ depending on $t_{\max }$. Thus, the expression $x^{-2} d \mu(x ; t)$ also determines a measure with the same support and the moments

$$
c_{-2}(t)=\int_{-\infty}^{\infty} \frac{d \mu(x ; t)}{x^{2}}, \quad c_{-1}(t)=\int_{-\infty}^{\infty} \frac{d \mu(x ; t)}{x}, \quad c_{0}, c_{1}, c_{2}, \ldots
$$

The function $m(z ; t)$ satisfies

$$
\begin{aligned}
m(z ; t) & =\int_{-\infty}^{\infty} \frac{1}{1-\frac{z}{x}} \cdot \frac{d \mu(x ; t)}{x}=\int_{-\infty}^{\infty} \sum_{k=0}^{\infty}\left(\frac{z}{x}\right)^{k} \cdot \frac{d \mu(x ; t)}{x} \\
& =\int_{-\infty}^{\infty} \frac{d \mu(x ; t)}{x}+z \int_{-\infty}^{\infty} \frac{d \mu(x ; t)}{x^{2}}+O\left(z^{2}\right) \\
& =c_{-1}(t)+z c_{-2}(t)+O\left(z^{2}\right) .
\end{aligned}
$$

The expression on the right-hand side coincides with the Taylor expansion of $m(z ; t)$ near the origin. Consequently,

$$
c_{-1}(t)=m(0 ; t) \quad \text { and } \quad c_{-2}(t)=\frac{d m}{d z}(0 ; t)
$$

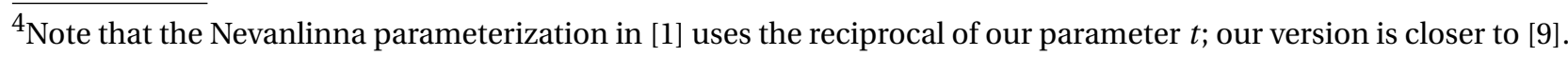


Then the equalities (7) yield

$$
\begin{aligned}
c_{-1}(t) & =-\frac{a(0)-t c(0)}{b(0)-t d(0)}=-\frac{0-t}{-1-0 t}=-t \text { and } \\
c_{-2}(t) & =-\frac{\left(a^{\prime}(0)-t c^{\prime}(0)\right)(b(0)-t d(0))-(a(0)-t c(0))\left(b^{\prime}(0)-t d^{\prime}(0)\right)}{(b(0)-t d(0))^{2}} \\
& =a^{\prime}(0)-t c^{\prime}(0)-t\left(b^{\prime}(0)-t d^{\prime}(0)\right)=t^{2} d^{\prime}(0)+a^{\prime}(0)-t\left(c^{\prime}(0)+b^{\prime}(0)\right) .
\end{aligned}
$$

Our consideration almost literally repeats [8, p. 181], we therefore can obtain that the moment sequence ( $\underline{8})$ is definite in the same way. Let us however relate it to M. Riesz's Theorem on $\mathrm{N}$-extremality and completeness of orthogonal polynomials, see e.g. [2, p. 2796, p. 2801] or [1, p. 55]. Let $\left(P_{k}(z)\right)_{k=0}^{\infty}$ be the sequence of the orthonormal polynomials induced by $\mathfrak{h}$ or, which is the same, by $d \mu(x ; t)$ for any $t \in\left(-t_{\max }, t_{\max }\right)$; let $\left(Q_{k}(z)\right)_{k=0}^{\infty}$ denote the corresponding polynomials of the second kind. Given any non-real number $z$, Parseval's equality for $(x-z)^{-1}$ has the form:

$$
\frac{m(z ; t)-m(\bar{z} ; t)}{z-\bar{z}}=\int_{-\infty}^{\infty} \frac{d \mu(x ; t)}{|x-z|^{2}}=\sum_{k=0}^{\infty}\left|m(z ; t) P_{k}(z)+Q_{k}(z)\right|^{2},
$$

see $[1$, p. 40]. The series on the right hand side converge due to the indeterminacy of $\mathfrak{h}$. The limit of this equality as $z \rightarrow 0$ exists due to $0 \notin \operatorname{supp} d \mu(x ; t)$; it is equal to

$$
\begin{aligned}
c_{-2}(t)=\frac{d m}{d z}(0 ; t) & =\sum_{k=0}^{\infty}\left|m(0 ; t) P_{k}(0)+Q_{k}(0)\right|^{2} \\
& =c_{-1}^{2}(t) \sum_{k=0}^{\infty} P_{k}^{2}(0)+\sum_{k=0}^{\infty} Q_{k}^{2}(0)+2 c_{-1}(t) \sum_{k=0}^{\infty} P_{k}(0) Q_{k}(0),
\end{aligned}
$$

which is another form of (10), cf. [1, p. 54]. By ( $\underline{9})$, each value of $c_{-1}(t)$ corresponds to a single $\mathrm{N}$-extremal measure $d \mu(x ; t)$. At the same time, the choice of $c_{-2}(t)$ as in $(10)$ is equivalent to Parseval's equality and, hence, to the $\mathrm{N}$-extremality. ${ }^{5}$ So, the moment sequence (8) determines a unique measure. If a feasible choice of $c_{-1}$ and $c_{-2}$ does not fix a unique measure, then Parseval's equality turns to Bessel's inequality (므).

More specifically, let $c_{-1}$ and $c_{-2}$ be given real numbers. If the equality in (5) holds, then $c_{-2}=$ $c_{-2}\left(-c_{-1}\right)$ by (10) and, hence, the sequence $t$ is determinate. Suppose that $c_{-2}$ is an arbitrary real number satisfying the strict inequality in (5). Thus, there exists $\varepsilon>0$ such that both points $\left(c_{-1}, c_{-2}\right)$ and $\left(c_{-1}, c_{-2}-\varepsilon\right)$ lie strictly inside the parabolic region determined by (5). Then the quadratic equation

$$
t^{2} \sum_{k=0}^{\infty} P_{k}^{2}(0)-2 t \sum_{k=0}^{\infty} P_{k}(0) Q_{k}(0)+\sum_{k=0}^{\infty} Q_{k}^{2}(0)=c_{-2}
$$

has two distinct real solutions, say $t_{1}, t_{2}$. The same is true for the equation

$$
t^{2} \sum_{k=0}^{\infty} P_{k}^{2}(0)-2 t \sum_{k=0}^{\infty} P_{k}(0) Q_{k}(0)+\sum_{k=0}^{\infty} Q_{k}^{2}(0)=c_{-2}-\varepsilon,
$$

whose solutions we denote by $t_{3}, t_{4}$. Note that the points $\left(t_{1}, c_{-2}\right)$ and $\left(t_{2}, c_{-2}\right)$, as well as $\left(t_{3}, c_{-2}-\varepsilon\right)$ and $\left(t_{4}, c_{-2}-\varepsilon\right)$, are on the boundary of the above parabolic region. Since this region is convex, there exist $\vartheta, \eta \in(0,1)$ such that $c_{-1}=\vartheta t_{1}+(1-\vartheta) t_{2}=\eta t_{3}+(1-\eta) t_{4}$. Then the measure

$$
d v_{1}(x):=\vartheta \frac{d \mu\left(x ; t_{1}\right)}{x^{2}}+(1-\vartheta) \frac{d \mu\left(x ; t_{2}\right)}{x^{2}}
$$

\footnotetext{
${ }^{5}$ Under our conditions, Parseval's equality at the origin implies Parseval's equality everywhere outside the real line by M. Riesz's Theorem [1, p. 43]: its proof extends to the origin with minimal changes if $0 \notin \operatorname{supp} d \mu(x ; t)$.
} 
has the following moments:

$$
\begin{aligned}
\int_{-\infty}^{\infty} d v_{1}(x)=\vartheta c_{-2}\left(t_{1}\right)+(1-\vartheta) c_{-2}\left(t_{2}\right)=c_{-2}\left(t_{1}\right) & =c_{-2}, \\
\int_{-\infty}^{\infty} x d v_{1}(x)=\theta t_{1}+(1-\theta) t_{2} & =c_{-1}, \\
\theta c_{k}+(1-\theta) c_{k} & =c_{k} \quad \text { for } k=0,1,2, \ldots
\end{aligned}
$$

The measure

$$
d v_{2}(x):=\eta \frac{d \mu\left(x ; t_{3}\right)}{x^{2}}+(1-\eta) \frac{d \mu\left(x ; t_{4}\right)}{x^{2}}+\varepsilon \delta(x) d x
$$

in turn has the same sequence of moments:

$$
\begin{aligned}
\eta c_{-2}\left(t_{3}\right)+(1-\eta) c_{-2}\left(t_{4}\right)+\varepsilon=c_{-2}-\varepsilon+\varepsilon & =c_{-2}, \\
\eta t_{3}+(1-\eta) t_{4} & =c_{-1}, \\
\eta c_{k}+(1-\eta) c_{k} & =c_{k} \text { for } k=0,1,2, \ldots
\end{aligned}
$$

However, the supports of $d v_{1}(x)$ and $d v_{2}(x)$ have no common points, see e.g. the footnote in [1, p. 55]. Thus, the moment sequence $\mathfrak{t}=\left(c_{-2}, c_{-1}, c_{0}, c_{1}, \ldots\right)$ is indeterminate.

Proof of Theorem 6 . Since $\mathfrak{h} \in \operatorname{Indet}_{H}$ or $\operatorname{ind}_{0}(\mathfrak{h})<n$, the sequence $\left(c_{i}\right)_{i=2 n}^{\infty}$ is indeterminate. Then there is a corresponding $\mathrm{N}$-extremal measure, which has the atom $\varepsilon \delta(x) d x$ at the origin. Thus, each sequence $\left(c_{2 n}^{*}, c_{2 n+1}, \ldots\right)$ belongs to Indet ${ }_{H}$ provided that the positive number $c_{2 n}^{*}$ satisfies $\mid c_{2 n}^{*}-$ $c_{2 n} \mid<\varepsilon$.

Lemma 12 implies that, for an arbitrary real number $c_{2 n-1}^{*}$, there exists some $b_{2 n-2}$ such that

$$
\left(c_{2 n-2}^{*}, c_{2 n-1}^{*}, c_{2 n}^{*}, c_{2 n+1}, \ldots\right) \in \operatorname{Indet}_{H} \text { as soon as } c_{2 n-2}^{*}>b_{2 n-2} \text {. }
$$

Clearly, we can fix $c_{2 n-2}^{*}$ so that additionally $c_{2 n-2}^{*} \neq c_{2 n-2}$. Analogously, if we already have

$$
\left(c_{2 n-2 k}^{*}, \ldots, c_{2 n-1}^{*}, c_{2 n}^{*}, c_{2 n+1}, \ldots\right) \in \operatorname{Indet}_{H}
$$

for some integer $k, 1 \leq k<n$, then Lemma 12 yields that for an arbitrary real number $c_{2 n-k-1}^{*}$ there exists some $b_{2 n-2 k-2}$ such that

$$
\left(c_{2 n-2 k-2}^{*}, \ldots, c_{2 n-1}^{*}, c_{2 n}^{*}, c_{2 n+1}, \ldots\right) \in \operatorname{Indet}_{H} \text { for } c_{2 n-2 k-2}^{*}>b_{2 n-2 k-2} .
$$

By induction, the theorem is therefore true.

Proof of Theorem 10. The statement of this theorem only uses the "distance" between those sequences that differ in a finite number of entries. Since all norms in $\mathbb{R}^{n}$ are equivalent, it is enough to prove the theorem for the $l_{\infty}$ norm, i.e. the supremum of absolute values of the entries.

Let $n+1$ be the number of leading entries in an indeterminate Hamburger moment sequence $\mathfrak{h}$, that vary. To prove by induction on $n$, consider firstly the base case $n=0$. Since $\mathfrak{h} \in$ Indet $_{H}$, there exists the corresponding $\mathrm{N}$-extremal measure $d \mu(x)$ with an atom $\varepsilon_{0} \delta(x) d x$ at the origin, $\varepsilon_{0}>$ 0 . Therefore, $d \mu(x)+\gamma \delta(x) d x$ is a positive measure with the moments $c_{0}+\gamma, c_{1}, c_{2}, \ldots$ provided that $-\varepsilon_{0}<\gamma<\varepsilon_{0}$. As a result, $\left(f_{0}, c_{1}, c_{2}, \ldots\right) \in \operatorname{Indet}_{H}$ if $\left|f_{0}-c_{0}\right|<\varepsilon_{0}$.

Now assume that the following property is satisfied for $n-1 \geq 0$ :

$$
\max _{0 \leq i<n}\left|f_{i}-c_{i}\right|<\varepsilon_{n-1} \Longrightarrow\left(f_{0}, f_{1}, \ldots, f_{n-1}, c_{n}, c_{n+1}, \ldots\right) \in \operatorname{Indet}_{H} .
$$

For the inductive step, we need to show that there exists a number $\varepsilon_{n}>0$ such that

$$
\max _{0 \leq i<n+1}\left|f_{i}-c_{i}\right|<\varepsilon_{n} \Longrightarrow\left(f_{0}, f_{1}, \ldots, f_{n-1}, f_{n}, c_{n+1}, c_{n+2}, \ldots\right) \in \operatorname{Indet}_{H} .
$$


Recall that $\mathfrak{h} \in \operatorname{Indet}_{H}$. For certain small enough $\varepsilon>0$, if we take $d_{n}:=c_{n}+\varepsilon$ and $e_{n}:=c_{n}-\varepsilon$, then by Theorem 6 there exist real numbers $d_{0}, d_{1}, \ldots, d_{n-1}$ and $e_{0}, e_{1}, \ldots, e_{n-1}$ such that both

$$
\mathfrak{d}:=\left(d_{0}, d_{1}, \ldots, d_{n}, c_{n+1}, c_{n+2}, \ldots\right) \text { and } \mathfrak{e}:=\left(e_{0}, e_{1}, \ldots, e_{n}, c_{n+1}, c_{n+2}, \ldots\right)
$$

are indeterminate Hamburger moment sequences. Put

$$
\vartheta:=\frac{\varepsilon_{n-1} / 2}{\max _{0 \leq i<n} \max \left\{\left|d_{i}-c_{i}\right|,\left|e_{i}-c_{i}\right|, 1\right\}} \quad \text { and } \quad \varepsilon_{n}:=\min \left\{\frac{\varepsilon_{n-1}}{4}, \frac{\varepsilon}{2}\right\}
$$

and suppose that the real numbers $f_{0}, f_{1}, \ldots, f_{n}$ satisfy $\max _{0 \leq i<n+1}\left|f_{i}-c_{i}\right|<\varepsilon_{n}$.

Assume that $f_{n}<c_{n}$. Then the number

$$
\alpha:=\frac{c_{n}-f_{n}}{\vartheta\left(c_{n}-e_{n}\right)}<\frac{\varepsilon / 2}{\vartheta \varepsilon} \in\left[0, \frac{1}{2}\right)
$$

is such that

$$
f_{n}=c_{n}-\alpha \vartheta\left(c_{n}-e_{n}\right)=\alpha \vartheta e_{n}+(1-\alpha \vartheta) c_{n} .
$$

At the same time, the absolute value of $\zeta_{i}:=f_{i}-c_{i}+\alpha \vartheta\left(c_{i}-e_{i}\right)$ for $i<n$ satisfies

$$
\left|\zeta_{i}\right| \leq\left|f_{i}-c_{i}\right|+\alpha \vartheta\left|c_{i}-e_{i}\right|<\varepsilon_{n}+\frac{1}{2} \alpha \varepsilon_{n-1}<\frac{1}{4} \varepsilon_{n-1}+\frac{1}{4} \varepsilon_{n-1}=\frac{1}{2} \varepsilon_{n-1} .
$$

Consequently, for $i<n$

$$
\begin{aligned}
f_{i} & =c_{i}-\alpha \vartheta\left(c_{i}-e_{i}\right)+f_{i}-c_{i}+\alpha \vartheta\left(c_{i}-e_{i}\right) \\
& =\alpha \vartheta e_{i}+(1-\alpha \vartheta) c_{i}+\zeta_{i}=\alpha \vartheta e_{i}+(1-\alpha \vartheta)\left(c_{i}+\frac{\zeta_{i}}{1-\alpha \vartheta}\right) .
\end{aligned}
$$

Since

$$
\left|\frac{\zeta_{i}}{1-\alpha \vartheta}\right|<2 \cdot \frac{1}{2} \varepsilon_{n-1}=\varepsilon_{n-1},
$$

the sequence

$$
\mathfrak{g}:=\left(c_{0}+\frac{\zeta_{0}}{1-\alpha \vartheta}, c_{1}+\frac{\zeta_{1}}{1-\alpha \vartheta}, \ldots, c_{n-1}+\frac{\zeta_{n-1}}{1-\alpha \vartheta}, c_{n}, c_{n+1}, \ldots\right)
$$

in an indeterminate Hamburger moment sequence by the induction hypothesis. In other words, we obtainded the representation

$$
\left(f_{0}, f_{1}, \ldots, f_{n-1}, f_{n}, c_{n+1}, c_{n+2}, \ldots\right)=\alpha \vartheta \mathfrak{e}+(1-\alpha \vartheta) \mathfrak{g},
$$

where both terms on the right-hand side, and hence the left-hand side, belong to Indet ${ }_{H}$. This representation yields (11) in the case $f_{n}<c_{n}$. The case when $f_{n}>c_{n}$ follows analogously after replacing $\mathfrak{e}$ by $\mathfrak{d}$, so the theorem is therefore true for Hamburger moment sequences. The assertion on Stieltjes moment sequences follow from considering the related symmetric Hamburger sequence.

\section{Proof of Theorem 11}

Lemma 14. Suppose that $\mathfrak{h}=\left(c_{0}, c_{1}, c_{2}, \ldots\right)$ is a determinate Hamburger moment sequence and that a real number $\varepsilon>0$ and an integer $m>0$ are such that

$$
\mathfrak{t}:=\left(c_{0}, \ldots, c_{m-1}, c_{m}^{*}, c_{m+1} \ldots\right)
$$

is a Hamburger moment sequence for all $c_{m}^{*}$ satisfying $\left|c_{m}-c_{m}^{*}\right|<\varepsilon$. Then $\mathfrak{t}$ is also determinate and $\operatorname{ind}_{0}(\mathfrak{t})=\operatorname{ind}_{0}(\mathfrak{h})<\frac{m}{2}$. 
Proof. Denote $r:=\operatorname{ind}_{0}(\mathfrak{h})$ and $q=q\left(c_{m}^{*}\right):=\operatorname{ind}_{0}(\mathfrak{t})$. On the one hand, Theorem 4 implies that $2 r \leq$ $m$ and $2 q \leq m$. If $m$ is odd, both inequalities are clearly strict. Let us show that they are also strict on the assumption that $m$ is even. Indeed, for each fixed $c_{m}^{*} \in\left(c_{m}-\varepsilon, c_{m}+\varepsilon\right)$, the trimmed sequence

$$
\mathfrak{h}_{m}:=\left(c_{m}^{*}, c_{m+1}, c_{m+2}, \ldots\right)
$$

is either indeterminate or determinate of index 0 by the definition of index. The latter condition for a Hamburger sequence implies that the corresponding measure vanishes near the origin and adding a point mass at the origin makes it indeterminate (a straightforward consequence of e.g. Lemma 12). In other words, if $\mathfrak{h}_{m} \in \operatorname{Det}_{H}$ and its index is zero, then

$$
\left(c_{m}^{*}-\widetilde{\varepsilon}, c_{m+1}, c_{m+2}, \ldots\right)
$$

cannot be a Hamburger sequence when $\widetilde{\varepsilon}>0$. This however fails to be true for any $\widetilde{\varepsilon}<\varepsilon-\left(c_{m}-c_{m}^{*}\right)$; therefore, $\mathfrak{h}_{m} \in \operatorname{Indet}_{H}$ as soon as $\left|c_{m}-c_{m}^{*}\right|<\varepsilon$, which means $2 r<m$ and $2 q\left(c_{m}^{*}\right)<m$ for even $m$.

On the other hand, the trimmed sequence $\left(c_{i}\right)_{i=2 r}^{\infty}$ can be expressed as the following convex combination of two sequences:

$$
\begin{aligned}
\left(c_{2 r}, \ldots, c_{m-1}, c_{m}, c_{m+1} \ldots\right)= & \frac{1}{2}\left(c_{2 r}, \ldots, c_{m-1}, c_{m}^{*}, c_{m+1} \ldots\right) \\
& +\frac{1}{2}\left(c_{2 r}, \ldots, c_{m-1}, 2 c_{m}-c_{m}^{*}, c_{m+1}, \ldots\right) .
\end{aligned}
$$

Both sequences on the right-hand side are the Hamburger sequences provided that

$$
\left|c_{m}-\left(2 c_{m}-c_{m}^{*}\right)\right|=\left|c_{m}-c_{m}^{*}\right|<\varepsilon,
$$

so they are determinate, because their sum $\left(c_{i}\right)_{i=2 r}^{\infty}$ is determinate - see the explanation before Lemma 8. Consequently, $r \leq q\left(c_{m}^{*}\right)$.

Now, for each $c_{m}^{*} \in\left(c_{m}-\frac{\varepsilon}{2}, c_{m}+\frac{\varepsilon}{2}\right)$ the left-hand side of

$$
\begin{aligned}
2\left(c_{2 q}, \ldots, c_{m-1}, c_{m}^{*}, c_{m+1} \ldots\right)= & \left(c_{2 q}, \ldots, c_{m-1}, c_{m}, c_{m+1} \ldots\right) \\
& +\left(c_{2 q}, \ldots, c_{m-1}, 2 c_{m}^{*}-c_{m}, c_{2 m+1}, \ldots\right)
\end{aligned}
$$

is a determinate moment sequence. So, both sequences on its right-hand side must be determinate, and hence $q\left(c_{m}^{*}\right) \leq r$. Analogously, for each $c_{m}^{* *} \in\left(c_{m}^{*}-\frac{\varepsilon}{4}, c_{m}^{*}+\frac{\varepsilon}{4}\right)$ the identity

$$
\begin{aligned}
2\left(c_{2 q}, \ldots, c_{m-1}, c_{m}^{* *}, c_{m+1}, \ldots\right)= & \left(c_{2 q}, \ldots, c_{m-1}, c_{m}^{*}, c_{m+1}, \ldots\right) \\
& +\left(c_{2 q}, \ldots, c_{m-1}, 2 c_{m}^{* *}-c_{m}^{*}, c_{m+1}, \ldots\right)
\end{aligned}
$$

shows that both sequences on its right-hand side must be determinate, and hence $q\left(c_{m}^{* *}\right) \leq q\left(c_{m}^{*}\right) \leq$ $r$. In other words, we have $q\left(c_{m}^{*}\right) \leq r$ whenever $c_{m}^{*} \in\left(c_{m}-\frac{3}{4} \varepsilon, c_{m}+\frac{3}{4} \varepsilon\right)$. The same manipulations can be continued further, which yields the inequality $q\left(c_{m}^{*}\right) \leq r$ for all $c_{m}^{*} \in\left(c_{m}-\varepsilon, c_{m}+\varepsilon\right)$. It implies that $q=q\left(c_{m}^{*}\right)=r$ being combined with the reverse inequality $r \leq q\left(c_{m}^{*}\right)$ obtained above.

Lemma 15. No Hamburger moment sequence $\mathfrak{h} \in \operatorname{Det}_{H}$ with $\operatorname{ind}_{0}(\mathfrak{h})=0$ can satisfy the conditions of Lemma 14.

The proof of this lemma is based on relations between certain determinants of the corresponding Hankel matrix. More specifically, given a sequence $\mathfrak{e}=\left(e_{0}, e_{1}, e_{2}, \ldots\right)$ and two integer numbers $n>0$ and $k \geq 0$ denote

$$
\Delta_{n}^{(k)}[\mathfrak{e}]:=\left|\begin{array}{ccccc}
e_{2 k} & e_{2 k+1} & e_{2 k+2} & \ldots & e_{2 k+n-1} \\
e_{2 k+1} & e_{2 k+2} & e_{2 k+3} & \ldots & e_{2 k+n} \\
e_{2 k+2} & e_{2 k+3} & e_{2 k+4} & \ldots & e_{2 k+n} \\
\vdots & \vdots & \vdots & \ddots & \vdots \\
e_{2 k+n-1} & e_{2 k+n} & e_{2 k+n+1} & \ldots & e_{2 k+2 n-2}
\end{array}\right|
$$


If additionally $0 \leq i<k$ and $0 \leq j<k$, then

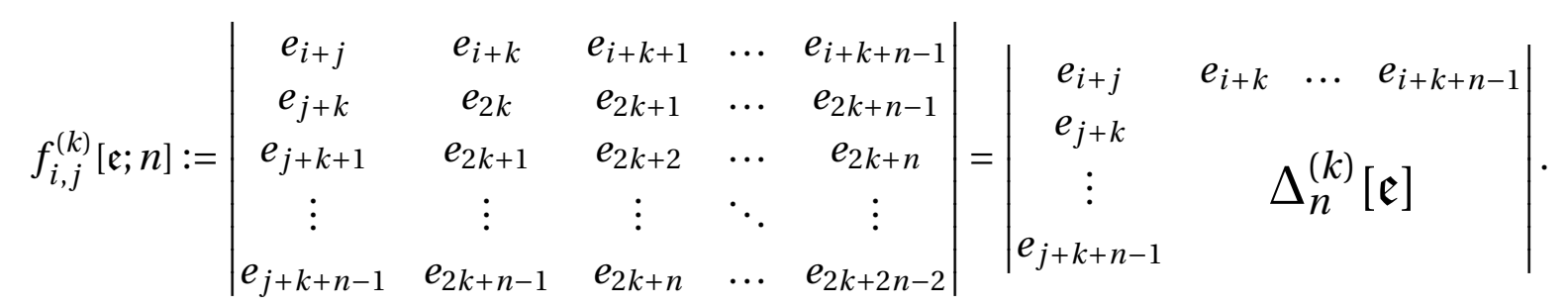

Since determinants are invariant under the transposition, the matrix

$$
F_{n}^{(k)}[\mathfrak{e}]:=\left(f_{i, j}^{(k)}[\mathfrak{e} ; n]\right)_{i, j=0}^{k-1}
$$

is symmetric.

Proof of Lemma 15. Put $\gamma:=c_{m}^{*}-c_{m}$ so that $c_{m}^{*}=c_{m}+\gamma$. Denote also

$$
f_{i, j}[\mathfrak{t}]:=f_{i, j}^{(m+1)}[\mathfrak{t} ; n-m-1]
$$

for the sake of brevity. The quadratic form

$$
\sum_{i, j=0}^{\infty} c_{i+j} x^{i} x^{j}+\gamma \sum_{k=0}^{m} x^{k} x^{m-k}
$$

corresponding to $\mathfrak{t}$ is positive definite, see Remark 2; hence, $\Delta_{n-m-1}^{(m+1)}[\mathfrak{t}]=\Delta_{n-m-1}^{(m+1)}[\mathfrak{h}]>0$. Sylvester's determinant identity [5, Chapter I, \$2] for the minors introduced above can be written as

$$
\Delta_{n}^{(0)}[\mathfrak{t}]=\left(\Delta_{n-m-1}^{(m+1)}[\mathfrak{t}]\right)^{-m} \cdot \operatorname{det} F_{n-m-1}^{(m+1)}[\mathfrak{t}] .
$$

On the right-hand side, only the entries $f_{k, m-k}[\mathfrak{t}]$ with $k=0,1, \ldots, m$ depend on $c_{m}^{*}$. More specifically,

$$
\begin{aligned}
& f_{k, m-k}[\mathfrak{t}]=\left|\begin{array}{cccc}
c_{m} & c_{k+m+1} & \ldots & c_{k+n-1} \\
c_{2 m-k+1} & & \\
\vdots & \Delta_{n-m-1}^{(m+1)}[\mathfrak{h}] \\
c_{m-k+n-1} &
\end{array}\right|+\left|\begin{array}{cccc}
\gamma & 0 & \ldots & 0 \\
c_{2 m-k+1} & & \\
\vdots & \Delta_{n-m-1}^{(m+1)}[\mathfrak{h}] \\
c_{m-k+n-1} & &
\end{array}\right| \\
& =f_{k, m-k}[\mathfrak{h}]+\gamma \Delta_{n-m-1}^{(m+1)}[\mathfrak{h}] .
\end{aligned}
$$

As a consequence,

$$
F_{n-m-1}^{(m+1)}[\mathfrak{t}]=\left(f_{i, j}[\mathfrak{t}]\right)_{i, j=0}^{m}=\left(f_{i, j}[\mathfrak{h}]+\gamma \delta_{i+j, m} \Delta_{n-m-1}^{(m+1)}[\mathfrak{h}]\right)_{i, j=0}^{m},
$$

where

$$
\delta_{i+j, m}= \begin{cases}0, & \text { if } i+j \neq m \\ 1, & \text { if } i+j=m\end{cases}
$$

is the Kronecker delta. The Hadamard inequality [5, Chapter I, §8] implies

$$
f_{i, i}[\mathfrak{h}] \leq c_{2 i} \cdot \Delta_{n-m-1}^{(m+1)}[\mathfrak{h}] \text { for } i=0,1, \ldots, m .
$$

All factors here are strictly positive as the principal minors of the Hankel matrix corresponding to $\underline{(12)}$ with $\gamma=0$. Moreover,

$$
\left|\begin{array}{ll}
f_{i, i}[\mathfrak{h}] & f_{i, j}[\mathfrak{h}] \\
f_{j, i}[\mathfrak{h}] & f_{j, j}[\mathfrak{h}]
\end{array}\right|=\Delta_{n-m-1}^{(m+1)}[\mathfrak{h}] \cdot\left|\begin{array}{ccccc}
c_{2 i} & c_{i+j} & c_{m+i} & \ldots & c_{i+n-1} \\
c_{i+j} & c_{2 j} & c_{m+j} & \ldots & c_{j+n-1} \\
c_{m+i} & c_{m+j} & \\
\vdots & \vdots & \Delta_{n-m-1}^{(m+1)}[\mathfrak{h}] \\
c_{i+n-1} & c_{j+n-1} &
\end{array}\right|>0
$$


for any $0 \leq i<j<m$ by Sylvester's determinant identity. Since the matrix $F_{n-m-1}^{(m+1)}[\mathfrak{h}]$ is symmetric, the last inequality can be rewritten with the help of (13) as

$$
\left(f_{i, j}[\mathfrak{h}]\right)^{2}<f_{i, i}[\mathfrak{h}] \cdot f_{j, j}[\mathfrak{h}] \leq c_{2 i} c_{2 j}\left(\Delta_{n-m-1}^{(m+1)}[\mathfrak{h}]\right)^{2} .
$$

Consequently, all entries of the matrix

$$
\left(\frac{f_{i, j}[\mathfrak{t}]}{\Delta_{n-m-1}^{(m+1)}[\mathfrak{t}]}\right)_{i, j=0}^{m}=\left(\frac{f_{i, j}[\mathfrak{h}]}{\Delta_{n-m-1}^{(m+1)}[\mathfrak{h}]}+\gamma \delta_{i+j, m}\right)_{i, j=0}^{m}
$$

are bounded in absolute value uniformly in $n$ and $\gamma \in(-\varepsilon, \varepsilon)$. Moreover, its determinant

$$
\begin{aligned}
p_{n}(\gamma) & :=\frac{\operatorname{det} F_{n-m-1}^{(m+1)}[\mathfrak{t}]}{\left(\Delta_{n-m-1}^{(m+1)}[\mathfrak{t}]\right)^{m+1}}=\operatorname{det}\left(\frac{f_{i, j}[\mathfrak{h}]}{\Delta_{n-m-1}^{(m+1)}[\mathfrak{h}]}+\gamma \delta_{i+j, m}\right)_{i, j=0}^{m} \\
& =(-1)^{\frac{m(m+1)}{2}} \gamma^{m+1}+\cdots+\frac{\operatorname{det} F_{n-m-1}^{(m+1)}[\mathfrak{h}]}{\left(\Delta_{n-m-1}^{(m+1)}[\mathfrak{h}]\right)^{m+1}} \gamma^{0} .
\end{aligned}
$$

is a polynomial in $\gamma$ of degree $m+1$. Its coefficients remain bounded uniformly in $n$, as certain sums of products of at most $m+1$ bounded factors.

The $m+2$-dimensional bounded set of coefficients of $p_{n}(\gamma)$ is necessarily compact. Therefore, there is a sequence $\left(n_{k}\right)_{k=1}^{\infty}$ such that the polynomials $p_{n_{k}}(\gamma)$ converge coefficientwise to

$$
p_{*}(\gamma)=(-1)^{\frac{m(m+1)}{2}} \gamma^{m+1}+\ldots
$$

In particular, $p_{*}(\gamma) \not \equiv 0$ due to the constant leading coefficient. The last condition however cannot be satisfied. Indeed, Lemma 14 yields that $\operatorname{ind}_{0}(\mathfrak{t})=\operatorname{ind}_{0}(\mathfrak{h})=0$. By Hamburger's criterion [8, pp. 183-185], this is equivalent to

$$
\lim _{n \rightarrow \infty} \frac{\Delta_{n}^{(0)}[\mathfrak{t}]}{\Delta_{n-1}^{(1)}[\mathfrak{t}]}=0 \quad \text { and } \quad \lim _{n \rightarrow \infty} \frac{\Delta_{n-k}^{(k)}[\mathfrak{t}]}{\Delta_{n-k-1}^{(k+1)}[\mathfrak{t}]}=\xi_{k}(\gamma), \quad 0<\xi_{k}(\gamma)<\infty, \quad k=1,2, \ldots,
$$

for some functions $\xi_{k}(\gamma)$ and all $\gamma \in(-\varepsilon, \varepsilon)$. The ratios are well defined, because the inequality $\Delta_{n-k}^{(k)}[\mathfrak{t}]>0$ follows from positive definiteness of the quadratic form (12). Consequently,

$$
0=\lim _{n \rightarrow \infty} \frac{\Delta_{n}^{(0)}[\mathfrak{t}]}{\Delta_{n-1}^{(1)}[\mathfrak{t}]}=\lim _{n \rightarrow \infty} \frac{\operatorname{det} F_{n-m-1}^{(m+1)}[\mathfrak{t}]}{\left(\Delta_{n-m-1}^{(m+1)}[\mathfrak{t}]\right)^{m} \Delta_{n-1}^{(1)}[\mathfrak{t}]}=\frac{1}{\prod_{k=1}^{m} \xi_{k}(\gamma)} \lim _{n \rightarrow \infty} \frac{\operatorname{det} F_{n-m-1}^{(m+1)}[\mathfrak{t}]}{\left(\Delta_{n-m-1}^{(m+1)}[\mathfrak{t}]\right)^{m+1}},
$$

that is $p_{*}(\gamma)=0$ for all $\gamma \in(-\varepsilon, \varepsilon)$ and, thus, $p_{*}(\gamma) \equiv 0$. This contradiction proves the lemma.

Proof of Theorem 11. The theorem's hypothesis for the Hamburger sequences reads: there exist some $\varepsilon>0$ and $m \geq 0$ such that

$$
\left(c_{0}, \ldots, c_{m-1}, c_{m}^{*}, c_{m+1} \ldots\right)
$$

is a Hamburger moment sequence for all $c_{m}^{*}$ satisfying $\left|c_{m}-c_{m}^{*}\right|<\varepsilon$. The case $m=0$ follows immediately from Hamburger's criterion of determinacy, so we assume $m>0$.

Let $\mathfrak{h}=\left(c_{0}, c_{1}, c_{2}, \ldots\right)$ be determinate, then Lemma 14 implies that $r:=\operatorname{ind}_{0}(\mathfrak{h})<\frac{m}{2}$. Therefore, the trimmed sequence

$$
\left(c_{2 r}, \ldots, c_{m-1}, c_{m}, c_{m+1}, \ldots\right)
$$

is determinate with the zero index by definition of the index. The last assertion however contradicts Lemma 15 , so $\mathfrak{h}$ cannot be determinate. 
Now, let $\varepsilon>0$ and let $\left(s_{0}, \ldots, s_{m-1}, s_{m}^{*}, s_{m+1} \ldots\right)$ be a Stieltjes moment sequence for all $s_{m}^{*}$ satisfying $\left|s_{m}-s_{m}^{*}\right|<\varepsilon$. Then

$$
\left(s_{0}, 0, \ldots, s_{m-1}, 0, s_{m}^{*}, 0, s_{m+1}, 0 \ldots\right)
$$

is a symmetric Hamburger moment sequence; it is indeterminate by the first part of Theorem 11 which is already proved. As a result, the correspondence between Stieltjes and symmetric moment sequences yields

$$
\left(s_{0}, \ldots, s_{m-1}, s_{m}, s_{m+1} \ldots\right) \in \operatorname{Indet}_{s} .
$$

\section{REFERENCES}

[1] Naum Il'ich Akhiezer, The classical moment problem and some related questions in analysis, Oliver \& Boyd, Edinburgh-London, 1965.

[2] Christian Berg and Antonio Durán, The index of determinacy for measures and the $l_{2}$-norm of orthonormal polynomials, Trans. Amer. Math. Soc. 347 (1995), no. 8, 2795-2811.

[3] Christian Berg and Macro Thill, Rotation invariant moment problems, Acta Math. 167 (1991), 207-227.

[4] Theodore Chihara, Indeterminate symmetric moment problems, J. Math. Anal. Appl. 85 (1982), no. 2, 331-346.

[5] F. R. Gantmacher and M. G. Krein, Oscillation Matrices and Kernels and Small Vibrations of Mechanical Systems, GITTL, Moscow, 1950 (Russian). English translation is published by: AMS Chelsea Publ., Providence (R.I.), 2002.

[6] Hans Hamburger, Über eine Erweiterung des Stieltjesschen Momentenproblems (Teil I), Mat. Ann., 81 (1920), 235319 (German).

[7] Hans Hamburger, Über eine Erweiterung des Stieltjesschen Momentenproblems (Teil II), Math. Ann. 82 (1920) 120-164 (German).

[8] Hans Hamburger, Über eine Erweiterung des Stieltjesschen Momentenproblems (Teil III), Math. Ann. 82 (1920) 168-187 (German).

[9] James Shohat and Jacob Tamarkin, The Problem of Moments, American Mathematical Society, New York, 1950. 